\title{
Large Scale Training through Spoken Tutorials to Promote and use OpenModelica
}

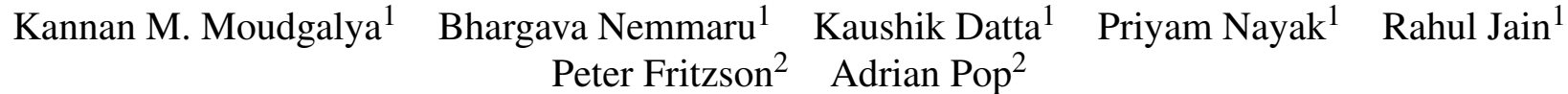 \\ ${ }^{1}$ Dept. of Chemical Engineering, Indian Institute of Technology Bombay, India, \\ kannan@iitb.ac.in, \{bnemmaru,kaushikdatta18, nayak.priyam22, rahjain1\}@gmail.com \\ ${ }^{2}$ Dept. Computer and Information Sciences, Linköping University, Sweden, \\ \{peter.fritzson, adrian.pop\} eliu.se
}

\begin{abstract}
The step-by-step self-teaching approach through audiovideo tutorials, known as Spoken Tutorials, has been very successful. About 3.4 million students in India have taken at least one course during the past 6-year period, of which 1.6 million students have attended the rapidly expanding course programme during 2016. This programme has now been expanded by a newly developed course in Modeling and Simulation with Modelica using the OpenModelica open source tool, primarily via the OMEdit graphical user interface. The spoken tutorial programme is exclusively based on free and open source software. This paper gives an introduction to the spoken tutorial approach and presents the recently developed spoken tutorial series for Modelica using OpenModelica. Feedback of participants shows that this series is an effective tool for self-learning of OpenModelica. The paper also presents a new web version that generalises the interactive DrModelica course material, OMWebbook: it enables students to learn Modelica, do text-based modeling exercises, and run simulations without needing to install a Modelica tool. OMWebbook is also planned to be covered in a future update to the spoken tutorial course on Modelica.

Keywords: Spoken Tutorial, tutorial, Modelica, OpenModelica, teaching, self learning, modelling, simulation
\end{abstract}

\section{Introduction}

Modelling and simulation is the most cost effective way for a developing country like India to become a developed one. For example, the warning issued by the Indian Space Research Organisation (ISRO) saved 10,000 lives recently (Laxman, 2016). It is well known that satellites help locate arable land, help predict locations in seas with fish population, etc. India's advances in the satellite technology are rooted in modelling and simulation. ISRO's interplanetary missions are said to be most cost and time effective, one of the reasons being the extensive use of simulation (EconomicTimes, 2013). Improving the modelling and simulation ethos amongst the academics can help derive such benefits in other sectors also.

Free and open source software (FOSS) is equally im- portant to a developing country like India. Use of proprietary software results in an outgo of $\$ 1$ billion every year, in education and police sectors alone (De, 2009). This precious foreign exchange can be more usefully spent in priority sectors like poverty alleviation and infrastructure development. Because of this, the Indian government has made the use of open source software mandatory, whenever it is of comparable capability to commercial software for the tasks at hand (Govt. of India, 2015). As OpenModelica implements the complete Modelica language (Modelica-Association, 2012) and is an open source implementation, we have selected it to promote the modelling and simulation culture in India. The award winning Spoken Tutorial method is selected for this promotion (Google, 2015; QS\&Wharton, 2015).

This paper is organised as follows. We begin with a brief explanation of the Spoken Tutorial methodology in sections 2 and 3. We list the Spoken Tutorials created on OpenModelica in section 4. We then explain in section 5 how to use these tutorials for effective learning without experts. In section 6, we explain the cloud environment OMWebbook In the final section, we conclude the paper with a discussion on future work.

\section{Spoken Tutorials}

A Spoken Tutorial is a ten minute long audio-video tutorial, created using the Screencast technology. It is well known that the optimal time for a video tutorial is about 9 minutes (Guo, 2013).

Here we use the tutorial concept in a slightly different way from a conventional 2-3 hour lecture-based tutorial. Spoken Tutorial is a smaller modular piece of active learning: A typical student following such an audio-video tutorial is expected to pause/replay the video and reproduce every command. To enable this, all the files used in the video are also seamlessly made available to the learner. As the software covered is open source, the student can download it, and practise the tutorial side-by-side. There are also exercises to perform. A beginner could easily spend half an hour to practise a 10 minute Spoken Tutorial, while an advanced learner may complete it in less time.

Spoken Tutorials are created to explain general IT con- 
cepts. Although only a small amount of information may be covered in ten minutes, one can cover advanced topics as well through a series of tutorials. The following factors distinguish this methodology:

1. Spoken Tutorials are created for self learning. We write the script before making the video. The script has to be certified as understandable by a beginner, before it is taken up for recording.

2. Spoken Tutorials are released through Creative Commons Attribution Share Alike (CC-BY-SA) license from our website (Spoken-Tutorial-Project, 2017b).

3. Spoken Tutorials are created on open source software only. As a result, anyone who wants to learn using Spoken Tutorial, can download and practise with the corresponding software.

4. We make available all the code, data files, etc., that are required in a tutorial, see Figure 6. This allows the learner to reproduce all the commands shown in the tutorial. This also allows a learner to start learning from any tutorial, obviating the need to go in a particular sequence.

5. We use the side-by-side method of learning through Spoken Tutorials. A schematic of the arrangement is given in Figure 7. This arrangement reduces the cognitive overload of learners (Moudgalya, 2014).

6. We dub the spoken part of Spoken Tutorials into all 22 official languages of India. For this, we time the script, which needs to be done only once. The fact that the video is in English helps from the employment perspective of our students. We have dubbed some of our tutorials also into some languages of the Middle East, South East Asia, Latin America and Africa. As our tutorials are available under the CCBY-SA license, these are available to everyone.

7. We have about 800 tutorials made into English, covering about 40 topics, such as, C, C++, Java, Python, PHP, Perl, Ruby, Scilab, LATEX, LibreOffice and DWSIM. We have about 5,600 dubbed tutorials. This is the largest collection of IT training material in Indian languages.

\section{Large Scale Training with Spoken Tutorials}

We now briefly explain the way we have used Spoken Tutorials to promote IT literacy:

1. We initially offered 2 hour free training to college students and faculty, to be done outside college hours. As Spoken Tutorials are created for self learning, presence of an expert is not necessary. Any interested person could organise a training session, using the resources at the college.
2. As it was difficult to find free slots, we mapped Spoken Tutorials to lab courses and encouraged learning during lab hours. As they do not have access to good teachers, most students in India find this a valuable resource that helps them understand the subject, score well in exams and get jobs.

3. We convinced university authorities, curriculum boards and syllabus committees to use Spoken Tutorials officially in their lab courses. More than 100 universities, each of which has about 50 to 500 affiliating colleges, have accepted the Spoken Tutorial methodologies, and have sent circulars recommending the use. At present, there are about 20,000 semester long lab courses that officially use Spoken Tutorials.

4. We now insist that we work only with colleges that agree to train ALL their students through Spoken Tutorials. This and the previous point have helped us train a really large number of students in the first full year of implementation, which is 2016, see Figure 1. We have trained a total of 2.8 million people in the past five years, reaching 1.6 million in 2016 alone. 200,000 people have already enrolled for our training during the first 20 days of this calendar year.

5. We provide online tests and certificates to college students who pass them. These are offered free of cost, thanks to the funding from our government.

6. Our website (Spoken-Tutorial-Project, 2017b) also receives a large number of visitors. In Figure 2, we present the statistics of visitors to our web page. It should be noted that most of our learners using the offline learning material, created using the facility described in Figure 4, which has increased the number of learners 50 times, as reported in Figure 2.

7. The web statistics are good from the view of other parameters as well. For example, the average time one spends on our web page (Spoken-TutorialProject, 2017b) is about 10 minutes and the bounce rate is about $30 \%$, as can be seen from Figure 3 .

We can use the procedure presented in this section to teach and thereby promoting OpenModelica and the Modelica language.

\section{OpenModelica Spoken Tutorials}

We created ten Spoken Tutorials on OpenModelica, as shown in Table 1. In this Table, we have listed the title of these tutorials and their learning objectives. The average time of these tutorials is about 13 minutes, with the minimum of 8 minutes and a maximum of 15 minutes. Although these are the first set of tutorials we created on OpenModelica, we have numbered them from 4, because of reasons to be explained next. 
Number of students/teachers trained in their colleges/schools

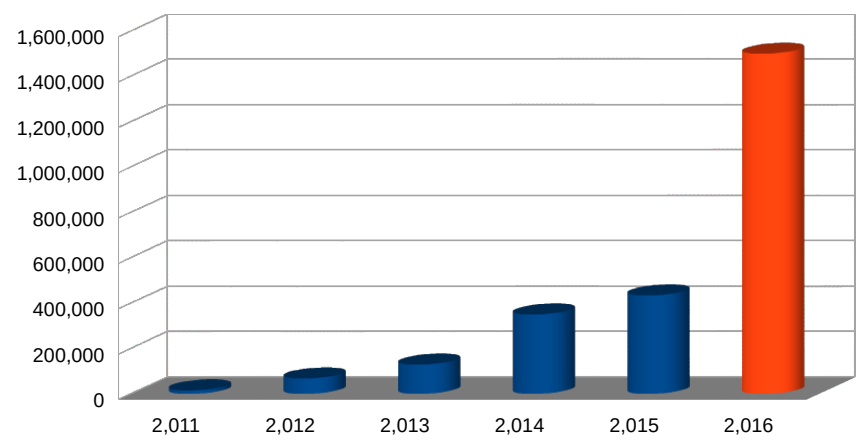

Figure 1. Growth of students/teachers trained. Our insistence that every student should be trained on at least one topic, and mapping to course content, have resulted in a large growth in 2016. The total number trained until now is about 3 million.

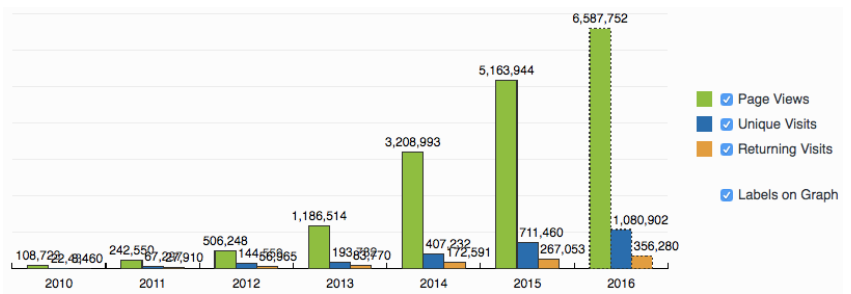

Figure 2. Growth of visits to ST website (Spoken-TutorialProject, 2017b). This can be obtained by visiting (StatCounter) and choosing Yearly data

In a recently held workshop (FOSSEE-Team, 2017), all the participants were asked to self-learn OpenModelica using Spoken Tutorials. We received the following feedback from the participants about the use of Spoken Tutorials:

- ... the detailed step-by-step descriptions made it easy to learn the basics of OpenModelica.

- Is is good for beginners. I suggest you to showcase more application use cases for the advanced use.

- ... very good, we have learned much from spoken tutorial.

- Spoken Tutorials are easy to Understand.

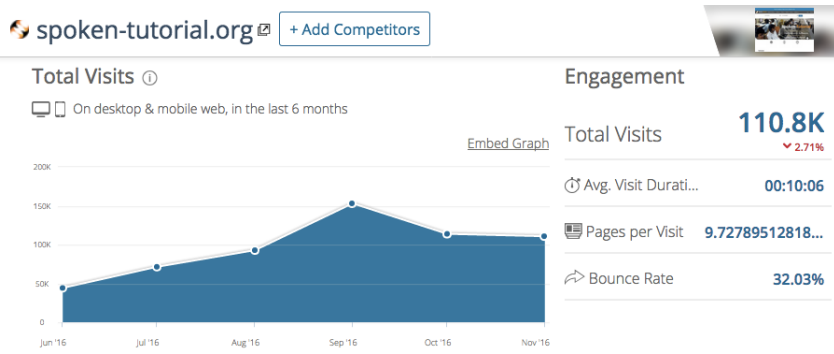

Figure 3. Analysis of visitor statistics of Spoken Tutorial, given by SimilarWeb (Team)
Table 1. Summary of Spoken Tutorials on OpenModelica

\begin{tabular}{|c|c|c|}
\hline No. & Name & Learning Objectives \\
\hline 4 & $\begin{array}{l}\text { Developing } \\
\text { an equa- } \\
\text { tion based } \\
\text { model }\end{array}$ & $\begin{array}{l}\text { Create a new Modelica class, vari- } \\
\text { ables, parameters and equations. } \\
\text { Assign initial, minimum and max- } \\
\text { imum values. Simulate model and } \\
\text { see results. }\end{array}$ \\
\hline 5 & $\begin{array}{l}\text { Control } \\
\text { flow and } \\
\text { event } \\
\text { handling }\end{array}$ & $\begin{array}{l}\text { Explain if-else statements, time } \\
\text { and state events, when statement, } \\
\text { and reinit function. }\end{array}$ \\
\hline 6 & $\begin{array}{l}\text { Functions } \\
\text { and types }\end{array}$ & $\begin{array}{l}\text { Define functions, data types, input } \\
\text { and output variables, assignment } \\
\text { statements, and the algorithm sec- } \\
\text { tion. Evaluate a polynomial. }\end{array}$ \\
\hline 7 & $\begin{array}{l}\text { Arrays in } \\
\text { Modelica }\end{array}$ & $\begin{array}{l}\text { Define vectors, vector indexing, } \\
\text { array variables, for and while } \\
\text { loops, and nested for loop. }\end{array}$ \\
\hline 8 & $\begin{array}{l}\text { Array func- } \\
\text { tions and } \\
\text { operations }\end{array}$ & $\begin{array}{l}\text { Explain OMShell, array construc- } \\
\text { tion function and perform arith- } \\
\text { metic operations on vectors and } \\
\text { matrices. }\end{array}$ \\
\hline 9 & $\begin{array}{l}\text { Modelica } \\
\text { Packages }\end{array}$ & $\begin{array}{l}\text { Create a package of classes, refer- } \\
\text { ence classes in a package, import } \\
\text { statement, and using Modelica li- } \\
\text { brary. }\end{array}$ \\
\hline 10 & $\begin{array}{l}\text { Annotations } \\
\text { in Modelica }\end{array}$ & $\begin{array}{l}\text { Specify an annotation and define } \\
\text { a record. Explain through exper- } \\
\text { iment, model and documentation } \\
\text { annotations. }\end{array}$ \\
\hline 11 & $\begin{array}{l}\text { Icon and } \\
\text { diagram } \\
\text { views }\end{array}$ & $\begin{array}{l}\text { Specify icon and diagram views } \\
\text { of a class, insert a polygon and } \\
\text { an ellipse in icon/diagram view. } \\
\text { Introduce coordinate system, grid } \\
\text { and components. Explain origin } \\
\text { and extent concepts, and line and } \\
\text { fill styles. }\end{array}$ \\
\hline 12 & $\begin{array}{l}\text { Component } \\
\text { oriented } \\
\text { modelling }\end{array}$ & $\begin{array}{l}\text { Instantiate classes. Define com- } \\
\text { ponent orientation, acausal con- } \\
\text { nectors, resistors, sources and the } \\
\text { ground. Connecting classes and } \\
\text { pins. }\end{array}$ \\
\hline 13 & $\begin{array}{l}\text { Block } \\
\text { component } \\
\text { modelling }\end{array}$ & $\begin{array}{l}\text { Define blocks and connect them. } \\
\text { Use MISO blocks and Modelica } \\
\text { libraries. Define RealInput and } \\
\text { RealOutput connectors. Instanti- } \\
\text { ate sum and product functions. }\end{array}$ \\
\hline
\end{tabular}

- ... up to some extent this tutorial make learning easier but we should make some more basic tutorial on OpenModelica.

- In my opinion side by side tutorials are the best way to start learning a new software. In order to master anything it will take a lot of practice of course but 
Table 2. Summary of New Spoken Tutorials on OpenModelica

\begin{tabular}{|l|l|l|}
\hline No. & Name & Learning Objectives \\
\hline 1 & $\begin{array}{l}\text { Introduction } \\
\text { to OMEdit }\end{array}$ & $\begin{array}{l}\text { Introduction to OM, OMEdit, } \\
\text { opening a class from libraries } \\
\text { browser, simulating it and see- } \\
\text { ing the results in plots. Explain } \\
\text { through a heat transfer example in } \\
\text { thermal library. }\end{array}$ \\
\hline 2 & $\begin{array}{l}\text { Examples } \\
\text { through } \\
\text { OMEdit }\end{array}$ & $\begin{array}{l}\text { Expose learners to models from } \\
\text { electrical and mechanics libraries, } \\
\text { using rectifier and double pendu- } \\
\text { lum classes. Simulate and see the } \\
\text { results in plots. }\end{array}$ \\
\hline 3 & $\begin{array}{l}\text { OM Con- } \\
\text { nectors }\end{array}$ & $\begin{array}{l}\text { Train how to compose exist- } \\
\text { ing objects to create a new cir- } \\
\text { cuit. Concept of connectors intro- } \\
\text { duced. Explain through resistor, } \\
\text { capacitor, inductor, voltage source } \\
\text { and ground. }\end{array}$ \\
\hline
\end{tabular}

having the basics right makes practice a lot easier.

- Tutorials are excellent. If you could include tutorials on popular extensions available on Modelica, it would be a great help. For example, the device drivers library.

Tutorials in Table 1 were created as per the pedagogy we use in classes: teach the underlying language first. But this is not necessarily the best way when it is offered for self learning. Students who depend on commercial simulators are used to the plug and play method of learning, which is possible in OpenModelica if one uses the predefined models that come with the distribution. This will also reduce the fear of first time users.

To address the above issue, we created two more tutorials. The first one explains how heat transfer modelling can be done. The second tutorial is concerned with examples from Electrical Engineering and Mechanical Engineering. These two tutorials are created for the absolute beginners, who would like to learn how to use the already available models.

We created one more tutorial to explain how to connect predefined models. Such a facility is generally available in commercial simulators. Introduction to this capability right at the beginning increases the value of the simulator in the opinion of the learner. In this tutorial, we show how to connect the preexisting blocks to build a new model. We now ask new learners to practise these tutorials, before starting with the ones in the previous section.

These new tutorials are listed in Table 2. Although created later, they are numbered from 1 , as we recommend these to be practised first, before proceeding to the ones listed in Table 1.

\section{Method to Use OpenModelica Spo- ken Tutorials}

We now explain how to use OpenModelica Spoken Tutorials. One should remember that our method should be such that any volunteer can conduct these workshops. As volunteers may not know answers to questions the learners may have, we insist on only reproducing what is shown in Spoken Tutorials: the learner does not know anything, so why not first do the things suggested in the tutorial? The pros and cons of this approach are summarised in (Moudgalya, 2011). We also offer a timed forum that will help the learner to go through the answers of previous questions and to ask new questions (Spoken-TutorialProject, 2017a).

The first thing to do is to create an offline version using the create the cdcontent utility, as shown in Figure 4 . Of course, this has to be done only by the organiser of the workshop. This will create a zip file, which has to be unzipped and copied on to every computer thereafter. We now summarise the recommended way to use Spoken Tutorials to conduct an OpenModelica self-learning workshop:

1. Open the file index.html using Firefox or Chrome. Internet Explorer may not work correctly. One gets the page as shown in Figure 5. From the url inside the red box in this figure, one can see that the learning content comes from the file system.

2. Listen to the side by side spoken tutorial that appears on this page. This tutorial explains how the learner has to use this method. After that, one has to Select Foss Category and then Select Language and then Submit - these options are enclosed in a green box in Figure 5.

3. The learner has to learn all the tutorials in the resulting play list one by one. From the play list, one can select any tutorial. If one scrolls down, one can see if any code file comes with that tutorial. In Figure 6, one can see where the code files are available.

4. Figure 7 shows how to open the Spoken Tutorial and the OpenModelica software side by side. Using the side-by-side method (Moudgalya, 2014), the learner has to reproduce every command described in the tutorial. The learner has to do the assignments also. As a result, the learner ends up spending a lot more time, even though the tutorial itself may only be of ten minute duration.

5. Using the method explained above, one has to learn learn all Spoken Tutorials in the play list.

The above given explanation is for the offline user. The same procedure will work for online use of Spoken Tutorials. 
In the six month time since we created Spoken Tutorials under discussion, we have trained more than 5,000 people on OpenModelica. As this is achieved through the self learning of already created Spoken Tutorials, there is a potential to train many more.

\section{Cloud Environment OMWebbook to Ease Learning of OpenModelica}

DrModelica (Lengquist-Sandelin et al., 2003) is an interactive electronic document for learning Modelica textbased modeling and simulation using the OpenModelica tool OMNotebook. It is structured like a book, with chapters, sections, model examples, exercises, formulae, and figures (Asghar et al., 2011). The recent versions of DrModelica contain most of the model examples in (Fritzson 2014) in an editable and executable form, including plots of simulated models. DrModelica is intended for self-learning and includes exercises with solutions, where the solutions are temporarily hidden while the student is working on a problem. Thus, it is extremely useful to everyone, and especially to the beginning learners of the Modelica language and the OpenModelica tool.

Recently we have developed a web-based version of OMNotebook, called OMWebbook (http: // omwebbook.openmodelica.org/) which enables editing models, running simulations, and doing plots in a web-page (Figure 8). The appearance is very similar to the OMNotebook. Thus, the students need not install any Modelica tool on their computer. They do not even need a computer, the exercises can be done using a phone or pad. OMWebbook communicates with a server that performs the actual simulation and plots. Naturally, those who do not have bandwidth to access the Internet can use OMNotebook.

OMNotebook, and also OMWebbook, have recently been enhanced with support for typesetting Mathematical formulae using $\mathrm{LTT}_{\mathrm{E}} \mathrm{X}$ commands (Asghar et al., 2011). These commands are associated with a formulae cell (Figure 9) but can be hidden (Figure 10) when editing is not needed. Figure 10 shows a small part of a chemical engineering course book in OMNotebook and OMWebbook with some formulae that have been typeset using this approach.

We propose to develop Spoken Tutorials on DrModelica, OMNotebook and OMWebbook in the near future. This will help beginners to quickly learn how to use these powerful tools and hence will further ease the learning of OpenModelica. Given that modelling and simulation are advanced topics for most students in India, we need all the tools to make them accessible.

\section{Conclusions and Future Work}

In this work, we have outlined a procedure to improve the modelling and simulation ethos in India and elsewhere through Spoken Tutorial enabled self-learning of OpenModelica. Based on the user feedback, we are in the process of increasing the offering of Spoken Tutorials on other OpenModelica topics. Through this effort, the powerful modelling paradigm of Modelica is expected to reach many people in India.

In the companion paper presented by our group in this conference (Jain et al., 2017), we have explained how the features of OpenModelica have been extended to make it useful to chemical engineers. We seek such contributors in other domains too. We hope to promote the use of the Modelica language and the OpenModelica tool with specific focus on different domains of application.

We also seek collaborators who would want to make available this important technology in their countries.

\section{Acknowledgements}

This work has been supported by Swedish Vinnova governmental agency and the Indian DST governmental agency in the Indo-Swedish RTISIM project, and by the National Mission on Education through ICT, Ministry of Human Resource Development, through the Spoken Tutorial project. The OpenModelica development is supported by the Open Source Modelica Consortium. 
| spoken-tutorial.org/cdcontent/

C Q Search

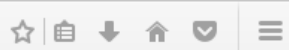

Spoken Tutorial so

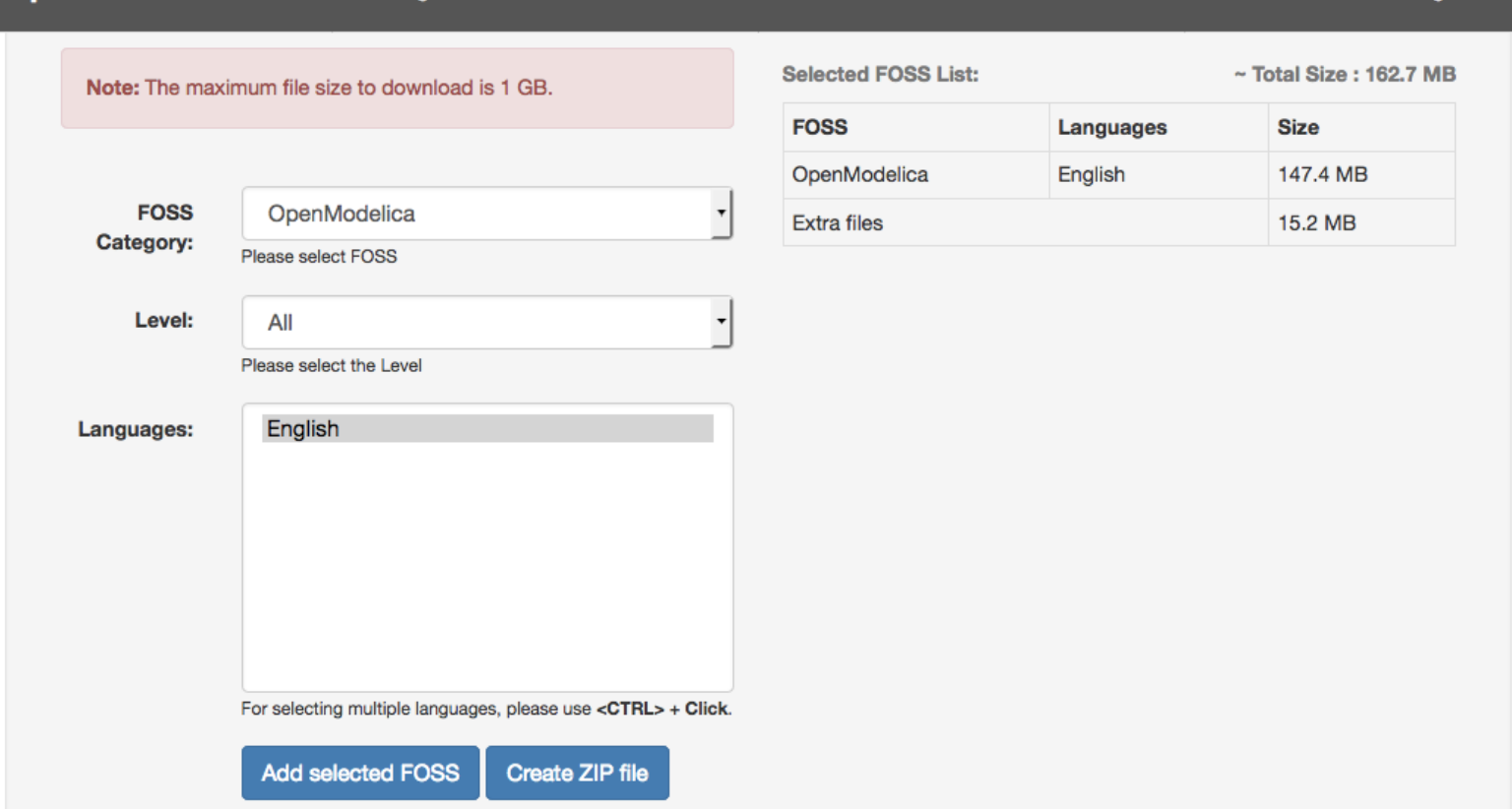

Figure 4. Creating a zip file of all tutorials for offline use, using the cdcontent facility

Q. Search Tutorials|spoken-t... $x \quad+$

file:///Users/kannan/Downloads/spoken/index.html

C Q Search

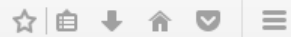

\section{Spoken Tutorial}

\section{:三 Search Tutorials}

Select Foss Category $\quad-\quad$ Select Language $\quad-1$ Submit

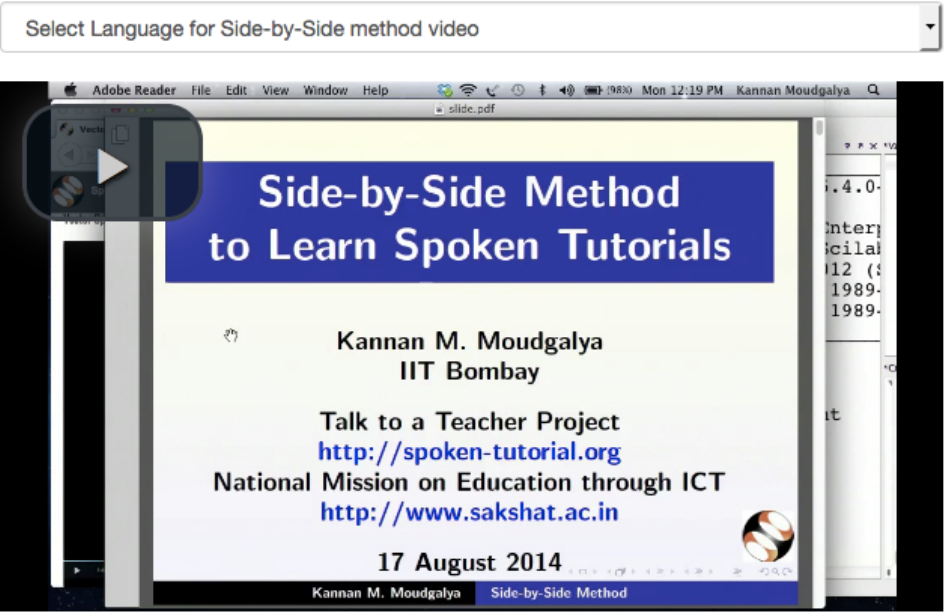

Figure 5. Unzipped content, opened in Firefox or Chrome. Internet Explorer may not work correctly. The URL inside the red box points to a file. One has to first listen to the side-by-side method tutorial. After that, one selects the FOSS, then the language, such as English, and then Submit. This will give a play list. One can then open any of the tutorials and practise side by side, as shown in 7 
(i) spoken-tutorial.org/watch/OpenModelica/Developing\%2Ban\%2Bequation-based\%2Bmodel/English/

C Q Search

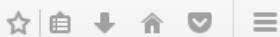

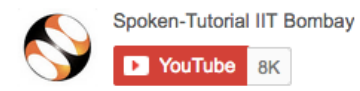

Instruction Sheet / Report Missing Component / Post your Question on the Forum

\section{Outline:}

Introduction to OMEdit

Declaration of variables and equations

Simulation of a model in OMEdit

Changing simulation conditions using 'Simulation Setup' toolbox
651 visits

Show video info

D Pre-requisite

No Pre-requisites for this tutorial.

Assignment

\section{Code files}

- Code Files/

- Code Files/freeFall.mo

\section{O Script}

Slides

- Slides/

- Slides/FOSSEE.jpeg

- Slides/ST-logo.jpeg

- Slides/developing-an-equation-

Figure 6. In the selected tutorial, one has to scroll down to locate the code files. Its location is indicated by the red oval in the above figure. Code files help the learner reproduce every command.

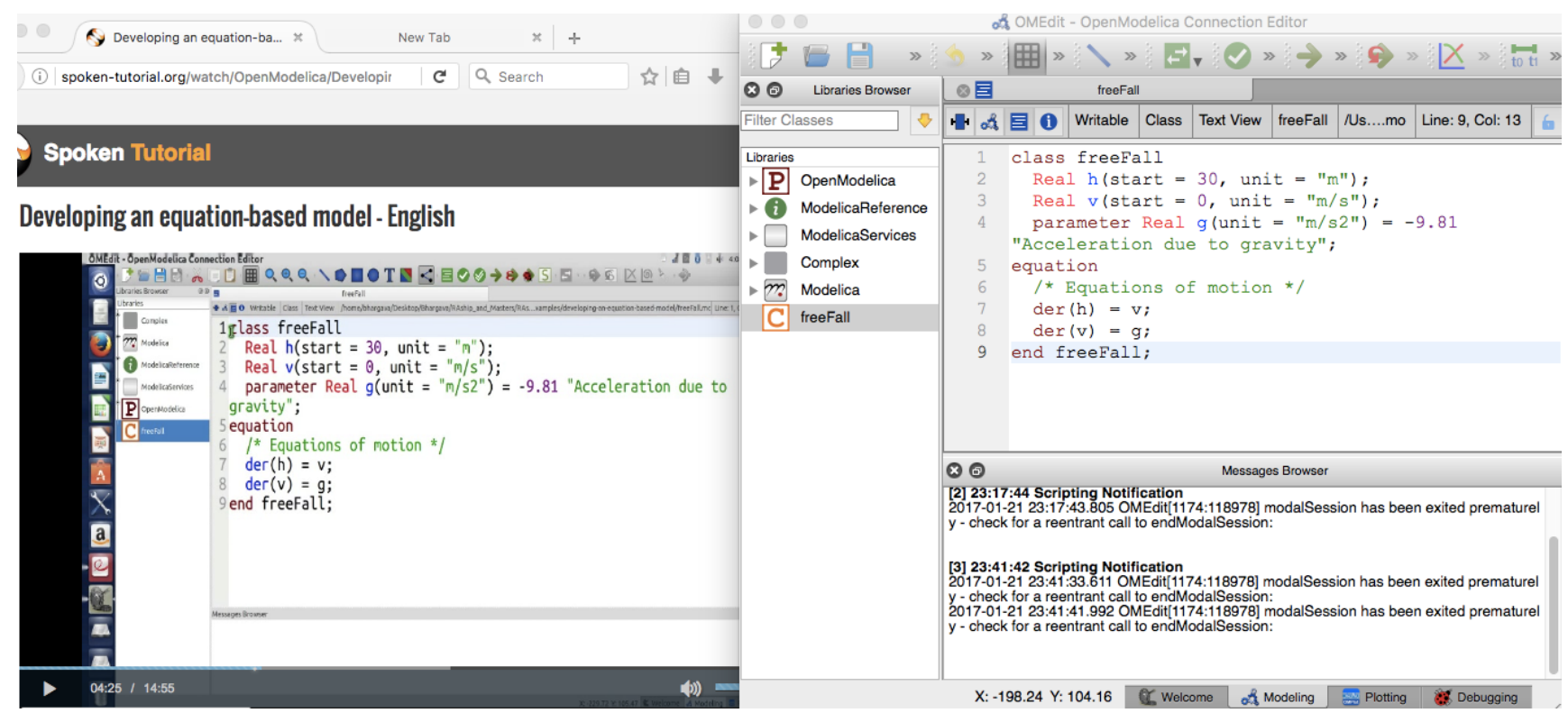

Figure 7. Side-by-side method. On the left hand side, we have the spoken tutorial, developing an equation based model. On the right hand side, OMEdit is displayed. The code file that came with the tutorial is opened in OMEdit. 


\begin{tabular}{|c|c|c|c|c|c|c|c|c|c|c|c|c|}
\hline \multicolumn{2}{|c|}{ OMWEBbook } & \multicolumn{4}{|l|}{$\times \quad+$} & \multirow[b]{2}{*}{ 出 } & \multirow[b]{2}{*}{ 白 } & \multirow[b]{2}{*}{$t$} & \multirow[b]{2}{*}{$\boldsymbol{\kappa}$} & \multirow[b]{2}{*}{$\boldsymbol{v}$} & - & ㅁ \\
\hline$\leftarrow$ & (i) omwebbor & copenmodelica.or! & ⿷匚] & $c$ & Q Search & & & & & & (aid - & $\gg$ \\
\hline & MWebBook & Evaluate Cell & Eval $A$ & & & & & & & & & \\
\hline
\end{tabular}

\section{First Basic Class}

\section{HelloWorld}

The program contains a declaration of a class called Helloworld with two fields and one equation. The first field is the variable $\mathrm{x}$ which is initialized to a start value 1 at the time when the simulation starts. The second field is the variable $a$, which is a constant that is initialized to 1 at the beginning of the simulation. Such a constant is prefixed by the keyword parameter in order to indicate that it is constant during simulation but is a model parameter that can be changed between simulations.

The Modelica program solves a trivial differential equation: $x^{\prime}=-a \star x$. The variable $x$ is a state variable that can change value over time. The $x^{\prime}$ is the time derivative of $x$.

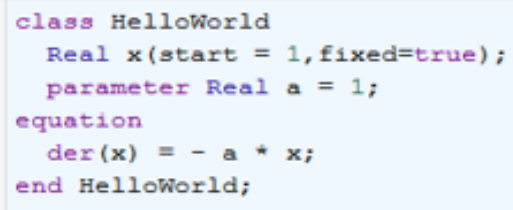

\section{Simulation of HelloWorld}

1 simulate ( Helloworld, startTime=0, stopTime=4)

2

Plot the results.

plot $(x)$

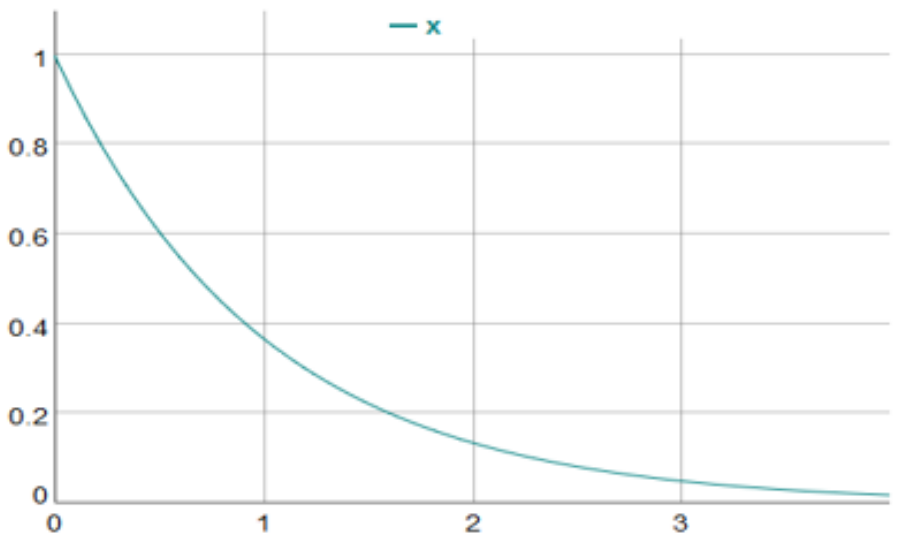

Figure 8. Example using OMWebbook on the simple HelloWorld model in DrModelica 


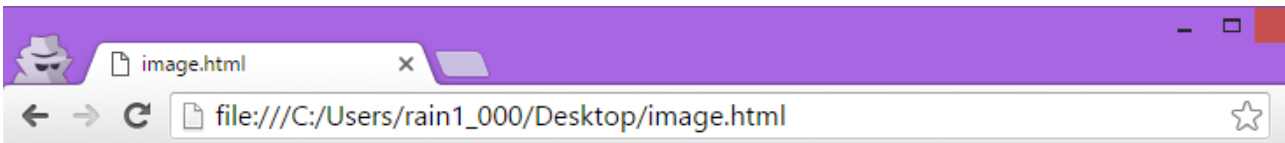

\section{Chemical Reactions}

When dealing with chemical reactions, molecules of one species of matter may react with the molecules of second species, thus forming a third species i.e., a different substance. This has a number of implications for $t]$ behavior of systems including chemical reactions. Traditionally, the literature of chemical reaction dynamics $h$ been divided into two main areas (not mentioning the large number of different application areas in chemistry)

1) Chemical reaction kinetics

2) Chemical thermodynamics

\section{Chemical Reaction Kinetics of Hydrogen Iodine}

A chemical reaction represented by a reaction formula transforms the chemical species on the left-hand side of the arrow, called reactants, to the species on the right-hand side of the arrow, called products: reactants $>$ products

Consider a chemical reaction between hydrogen gas and iodine gas to form hydrogen iodine

$\mathrm{H}_{2}+\mathrm{I}_{2} \leftrightarrow 2 \mathrm{HI}$

We can formulate the differential equations for the whole reaction system as below.

$$
\begin{gathered}
\frac{\mathrm{d}}{\mathrm{dt}}\left[H_{2}\right]=k_{2} \cdot\left[H I^{2}\right]-k_{1} \cdot\left[H_{2}\right] \cdot\left[I_{2}\right] \\
\frac{\mathrm{d}}{\mathrm{dt}}\left[I_{2}\right]=k_{2} \cdot\left[H I^{2}\right]-k_{1} \cdot\left[H_{2}\right] \cdot\left[I_{2}\right] \\
\frac{\mathrm{d}}{\mathrm{dt}}[H I]=2 k_{2} \cdot\left[H_{2}\right] \cdot\left[I_{2}\right]-2 k_{2} \cdot\left[H I^{2}\right]
\end{gathered}
$$

Figure 9. Type-setting mathematical formulae in OMNotebook using LTEX commands

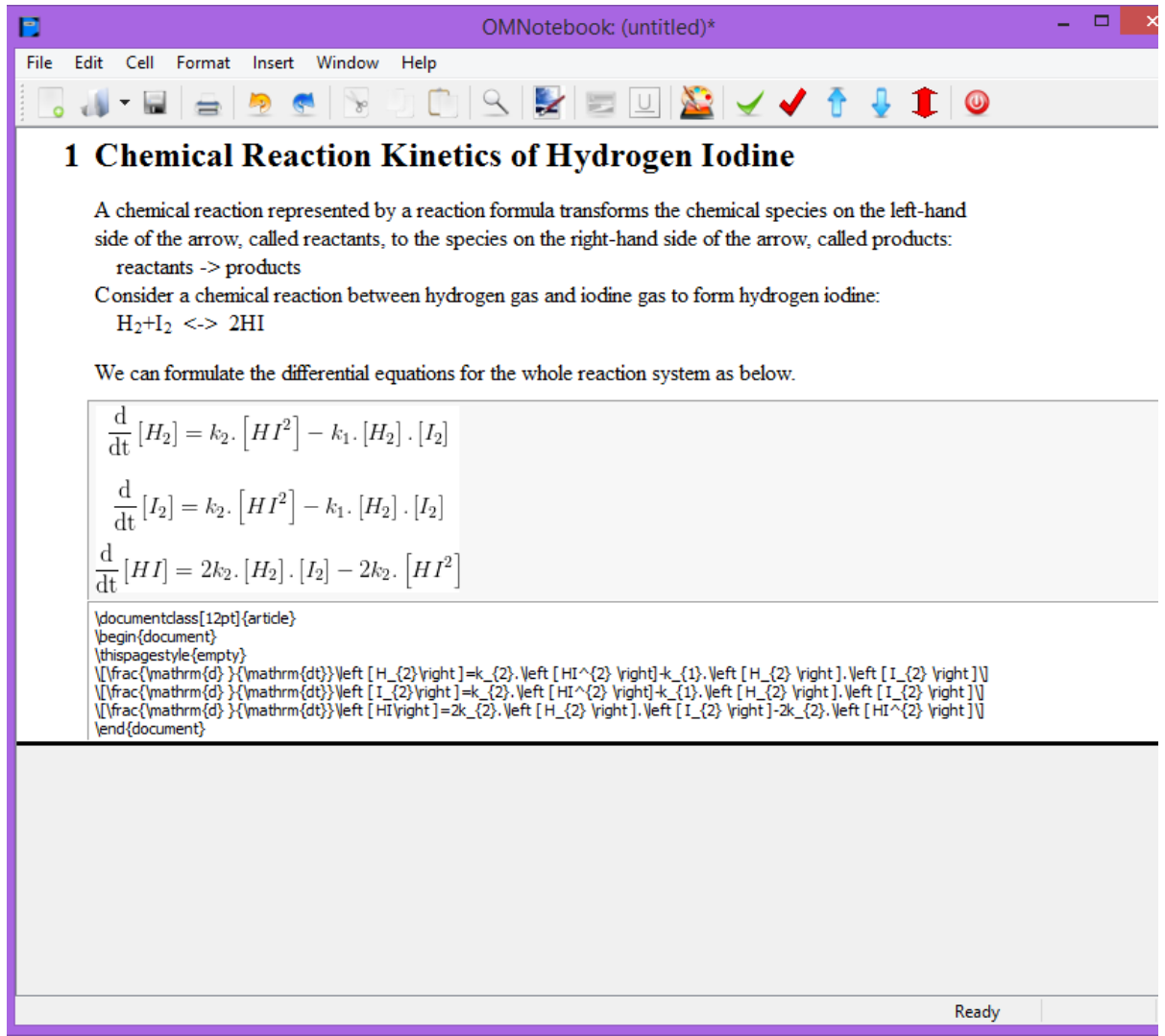

Figure 10. Example of finished typesetting of mathematical formulae in OMNotebook and OMWebbook 


\section{References}

A. Asghar, S. Tariq, M. Torabzadeh-Tari, P. Fritzson, A. Pop, M. Sjölund, P. Vasaiely, and W. Schamai. An Open Source Modelica Graphic Editor Integrated with Electronic Notebooks and Interactive Simulation. In Proc. of the 8th International Modelica Conference 2011, pages 739-747, Linköping university, Sweden, 2011.

R. De. Economic impact of free and open source software - a study in india. Technical Report http: / / www.iimb.ernet.in/ rahulde/ RD_FOSSRep2009.pdf, IIM Bangalore, 2009.

EconomicTimes. Why isro's mars mission is the cheapest. http://economictimes.indiatimes.com/ slideshows/science-technology/whyisros-mars-mission-is-the-cheapest/ harness-software-work-fast/slideshow/ $24982272 . \mathrm{cms}, 31$ Oct. 2013.

FOSSEE-Team. Openmodelica workshop. http:// fossee.in/workshop/om/, 4-5 Jan. 2017.

Google. Google mooc focused research awards. https: //research.googleblog.com/2015/ 03 /announcing-google-mooc-focusedresearch.html, March 2015. Last seen on 22 Jan. 2017.

Govt. of India. Policy on adoption of open source software for govt. of india. Gazette Notification, http://www.indianemployees.com/ gazette-notifications/details/policyon-adoption-of-open-source-software-forgovt-of-india/, 2015. English notification is given after that in Hindi. Last seen on 22 Jan. 2017.

P. Guo. Optimal Video Length for Student Engagement. See https://www.edx.org/blog/optimalvideo-length-student-engagement, 2013. Last seen on 3 April 2017.

R. Jain, K. M. Moudgalya, P. Fritzson, and A. Pop. Development of a Thermodynamic Engine in OpenModelica. In 12th Int. Modelica Conf., Prague, 2017. Modleica Association.

S. Laxman. Cyclone Vardah: ISRO satellites saved 10,000 lives in Tamil Nadu. Times of India, http: //timesofindia.indiatimes.com/india/ cyclone-vardah-isro-satellites-saved10000-lives-in-tamil-nadu/articleshow/ 55992320.cms, 17 December 2016. Last seen on 3 April 2017.

EL. Lengquist-Sandelin, S. Monemar, P. Fritzson, and P. Bunus. DrModelica - An Interactive Tutoring Environment for Modelica. In Proceedings of the 3rd International Modelica Conference, Linköping, Sweden, 3-4 Nov. 2003.

Modelica-Association. Modelica: A unified object-oriented language for physical systems modeling, language specification version 3.3. http: //www.modelica.org/, May 2012.

K. M. Moudgalya. LATEX Training through Spoken Tutorials. TUGboat, 32(3):251-257, 2011.

K. M. Moudgalya. Pedagogical and Organisational Issues in the Campaign for IT Literacy Through Spoken Tutorials. In R. Huang, Kinshuk, and N.-S. Chen, editors, The new development of technology enhanced learning, chapter 13, pages 223-244. Springer-Verlag, Berlin Heidelberg, 2014.

QS\&Wharton. Reimagine education 2015: Spoken Tutorial is Placed First in the Nurturing Employability Award Category. http: / / application.reimagine-education.com/ the-winners-individual/2015/132/ 2193b0ae3841f24da1464d4b6b70ee0f/Indian+ Institute+of+Technology+Bombayo22, Dec. 2015.

Spoken-Tutorial-Project. Online forum. See http:// forums.spoken-tutorial.org/, 2017a. Last seen on 3 April 2017.

Spoken-Tutorial-Project. Official web page. See http:// spoken-tutorial.org/, 2017b. Last seen on 3 April 2017.

StatCounter. Summary log. http: / / statcounter.com/ p5528933/summary/?account_id= $2904483 \&$ login id $=5 \&$ code $=$ 9f03e451b379437c7356d2529c726a7a\&guest_login= 1. Last seen on 12 Dec. 2016.

Similar Web Team. Get insights for any website or app. https : //www.similarweb.com/. Last seen on 12 Dec. 2016. 